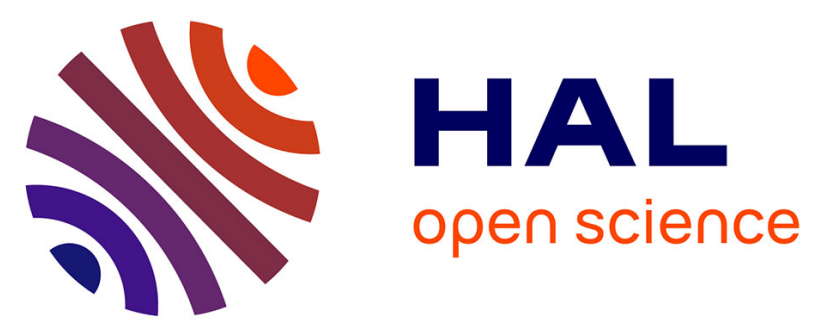

\title{
Propagation of three-dimensional bipolar ultrashort electromagnetic pulses in an inhomogeneous array of carbon nanotubes
}

Eduard Fedorov, Alexander Zhukov, Roland Bouffanais, Alexander

Timashkov, Boris Malomed, Hervé Leblond, Dumitru Mihalache, Nikolay

Rosanov, Mikhail Belonenko

\section{To cite this version:}

Eduard Fedorov, Alexander Zhukov, Roland Bouffanais, Alexander Timashkov, Boris Malomed, et al.. Propagation of three-dimensional bipolar ultrashort electromagnetic pulses in an inhomogeneous array of carbon nanotubes. Physical Review A, 2018, 97 (4), pp.043814. 10.1103/PhysRevA.97.043814 . hal-02572834

\section{HAL Id: hal-02572834 \\ https://univ-angers.hal.science/hal-02572834}

Submitted on 13 May 2020

HAL is a multi-disciplinary open access archive for the deposit and dissemination of scientific research documents, whether they are published or not. The documents may come from teaching and research institutions in France or abroad, or from public or private research centers.
L'archive ouverte pluridisciplinaire HAL, est destinée au dépôt et à la diffusion de documents scientifiques de niveau recherche, publiés ou non, émanant des établissements d'enseignement et de recherche français ou étrangers, des laboratoires publics ou privés. 


\title{
Propagation of three-dimensional bipolar ultrashort electromagnetic pulses in an inhomogeneous array of carbon nanotubes
}

\author{
Eduard G. Fedorov \\ Department of Biology, Technion-Israel Institute of Technology, Haifa 32000, Israel \\ and Vavilov State Optical Institute, 199053 Saint Petersburg, Russia \\ Alexander V. Zhukov \\ Singapore University of Technology and Design, 8 Somapah Road, 487372 Singapore \\ and Entropique Group Ltd., 3 Spylaw Street, Maori Hill, 9010 Dunedin, New Zealand \\ Roland Bouffanais \\ Singapore University of Technology and Design, 8 Somapah Road, 487372 Singapore
}

Alexander P. Timashkov

Saint Petersburg National Research University of Information Technologies, Mechanics and Optics (ITMO University), 197101 Saint Petersburg, Russia

Boris A. Malomed

Department of Physical Electronics, School of Electrical Engineering, Faculty of Engineering, Tel Aviv University, 69978 Tel Aviv, Israel and Saint Petersburg National Research University of Information Technologies, Mechanics and Optics (ITMO University),

197101 Saint Petersburg, Russia

Hervé Leblond

LUNAM Université, Université d'Angers, Laboratoire de Photonique d'Angers, EA 4464, 2 Boulevard Lavoisier, 49000 Angers, France

Dumitru Mihalache

Academy of Romanian Scientists, 54 Splaiul Independentei, Bucharest RO-050094, Romania and Horia Hulubei National Institute of Physics and Nuclear Engineering, Magurele RO-077125, Romania

Nikolay N. Rosanov

Vavilov State Optical Institute, 199053 Saint Petersburg, Russia;

Saint Petersburg National Research University of Information Technologies, Mechanics and Optics (ITMO University),

197101 Saint Petersburg, Russia;

and Ioffe Physical-Technical Institute, Russian Academy of Sciences, 194021 Saint Petersburg, Russia

Mikhail B. Belonenko

Laboratory of Nanotechnology, Volgograd Institute of Business, 400048 Volgograd, Russia;

Volgograd State University, 400062 Volgograd, Russia; and Entropique Group Ltd., 3 Spylaw Street, Maori Hill, 9010 Dunedin, New Zealand

(Received 1 February 2018; published 9 April 2018)

\begin{abstract}
We study the propagation of three-dimensional (3D) bipolar ultrashort electromagnetic pulses in an inhomogeneous array of semiconductor carbon nanotubes. The heterogeneity is represented by a planar region with an increased concentration of conduction electrons. The evolution of the electromagnetic field and electron concentration in the sample are governed by the Maxwell's equations and continuity equation. In particular, nonuniformity of the electromagnetic field along the axis of the nanotubes is taken into account. We demonstrate that depending on values of the parameters of the electromagnetic pulse approaching the region with the higher electron concentration, the pulse is either reflected from the region or passes it. Specifically, our simulations demonstrate that after interacting with the higher-concentration area, the pulse can propagate steadily, without significant spreading. The possibility of such ultrashort electromagnetic pulses propagating in arrays of carbon nanotubes over distances significantly exceeding characteristic dimensions of the pulses makes it possible to consider them as $3 \mathrm{D}$ solitons.
\end{abstract}

DOI: 10.1103/PhysRevA.97.043814 


\section{INTRODUCTION}

Nowadays, carbon nanotubes (CNTs)—quasi-onedimensional macromolecules of carbon-are considered as promising objects with a potential for applications to the development of the elemental base of modern electronics, including nanocircuits usable in neurocomputers [1]. Strong interest in these materials, starting from the moment of their discovery [2,3], is due to their unique physical properties (e.g., see Refs. [4-9]), which, in addition to the above-mentioned potential for the use in electronics, pave the way to a wide range of possibilities for the creation of ultrastrong composite materials, fuel cells, chemical sensors, and optical devices (such as displays, LEDs, and transparent conductive surfaces), etc. From the viewpoint of optoelectronic applications, specific features of the electronic structure of CNTs are of unique interest. The nonparabolicity of the dispersion law of conduction electrons (i.e., the energy dependence on the quasimomentum) in nanotubes makes it possible to observe a number of unique electromagnetic phenomena, including nonlinear diffraction and self-focusing of laser beams [10,11], as well as the propagation of solitary electromagnetic waves [12] at field strengths starting from $\sim 10^{3}-10^{4} \mathrm{~V} / \mathrm{cm}$. In this connection, it is relevant to mention that possibilities offered by modern laser technologies for the generation of powerful electromagnetic radiation with specified properties-including ultrashort laser pulses with the duration of the order of several half cycles of field oscillations $[13,14]$ - have stimulated studies of the propagation of electromagnetic waves in various novel media [15-25], with CNTs being one of them.

The possibility of propagation of solitary electromagnetic waves in arrays of CNTs has been first theoretically established in a one-dimensional (1D) model based on an assumption of uniformity of the field along the nanotube axis [12]. Actually, this approximation is only valid in a very narrow range of values of the underlying parameters. Subsequent studies have aimed at investigating increasingly more realistic models describing the evolution of the electromagnetic field in nanotube arrays, taking into regard various physical factors affecting the dynamics of the electromagnetic wave. Adding the complexity to the model's framework has proceeded in several directions: (i) increasing the dimensionality of the setting, (ii) taking into account nonuniformity of the electromagnetic wave field, and (iii) including various inhomogeneities of the medium. The investigation of these factors was carried out both independently, to clarify the role of each factor, and, subsequently, considering the combined effects of multiple factors.

Naturally, the first factor considered to make the model more realistic was the dimensionality of the model. The propagation of electromagnetic waves in arrays of CNTs was studied in the framework of 1D [12,26], 2D [27-30], and 3D [31,32] models. It was established that bipolar ultrashort electromagnetic pulses propagate, in a stable fashion, in the form of "breatherlike" light bullets over distances far exceeding the characteristic size of the pulses along the direction of their motion. The topicality of studying the propagation of electromagnetic waves in arrays of nanotubes in the inhomogeneous model is due, in particular, to the need to take transverse diffraction into account. Moreover, as in reality, a solitary electromagnetic wave in a real physical system is bounded in all directions, which implies nonuniformity of the field in any direction. Thus, the necessity of constructing a 3D model for the evolution of the electromagnetic field is obvious. In particular, nonuniformity of the field of the electromagnetic pulse along the axis of the nanotubes is an inherent ingredient of the 3D model. The construction of a model involving the latter feature was first reported in Refs. [30-32]. In particular, the propagation of ultrashort pulses in homogeneous arrays of CNTs has been investigated in 2D [30] and 3D [31] models. One of the main results of these studies is the prediction of redistribution of conduction electrons, leading to specific variations of the density of conduction electrons. Among possible practical applications of this phenomenon in microand nanoelectronics, one can envisage the manufacturing of highly accurate chemical sensors [31] based on specifically designed arrays of semiconductor CNTs. As shown in Ref. [32], such dynamical inhomogeneities in the electron subsystem of nanotubes can also underlie a complex medium-mediated mechanism of interaction of colliding electromagnetic pulses in the array of nanotubes.

Among factors that significantly affect the evolution of electromagnetic waves in arrays of semiconductor CNTs, a noteworthy one is the presence of various impurities and inhomogeneities. In Ref. [33], the propagation of a bipolar electromagnetic pulse in the $2 \mathrm{D}$ geometry, and in the presence of a multilevel impurity uniformly distributed over the sample, was investigated. It was shown that doping the medium with an impurity of this type leads to a modification of the characteristics of the propagating pulse as compared to the case of propagation in a "pure" sample.

The specific sample-doping format deserve particular consideration - in particular, localized introduction of impurities, limited to a certain part of the sample's volume, which implies the creation of an inhomogeneity. Such defects of the medium, which are not initially associated with the action of the electromagnetic field, can be, for example, implemented as metallic inclusions or layers containing an increased concentration of conduction electrons relative to the concentration in the homogeneous part of the sample. This kind of heterogeneity can emerge either as a consequence of technological failures, at the stage of sample manufacturing, or as a result of purposeful formation of the inhomogeneity with intended properties. The features of the interaction of 2D unipolar "light bullets" with metallic inclusions in nanotube arrays have been theoretically studied in Refs. [34,35]. The interaction of a bipolar ultrashort electromagnetic pulse with a layer of an increased electron concentration in the $2 \mathrm{D}$ model was studied both under the assumption of the uniformity of the field along the nanotube axis [36] and also taking into account nonuniformity of the field [37]. As a result, the selective nature of the interaction of the pulse with the static inhomogeneity of the medium has been established: a decrease of the pulse's duration and increase of its amplitude facilitates its passage through a layer of an increased electron concentration, while pulses with a longer duration and smaller amplitude can be reflected by this layer.

Thus, the propagation of electromagnetic pulses in inhomogeneous media-taking into account the nonuniformity of the pulses' field-are of significant interest. Indeed, there is a need to search for physical effects that can be used 
for the development of new components of the elemental base of optoelectronics, developing schemes for optical information processing and nondestructive testing systems, etc. Some peculiarities of light-matter interaction mentioned above may be employed for the development of ultrafast optical transistors, switches, logic elements, transmission and signal delay lines, and soliton memory elements [38], using ultrashort pulses as bits of data. Different outcomes of data processing operations may be associated with different regimes of the pulse propagation in the medium (e.g., different outcomes of the collision of a pulse with a layer of increased conductivity, embedded in the bulk of a semiconductor structure). It is therefore topical to implement such concepts using some of the most promising available materials, such as, in particular, graphene-based materials. In this connection, it is relevant to address effects of the static inhomogeneity in the electron subsystem of the array of semiconductor CNTs on the propagation of an ultrashort bipolar electromagnetic pulses in the most realistic 3D geometry. In this work, we consistently take into account factors that affect the dynamics of the ultrashort laser pulses, generalizing previously considered particular cases in the framework of an integrated model.

\section{THE SYSTEM'S CONFIGURATION AND KEY ASSUMPTIONS}

We consider the propagation of a solitary electromagnetic wave in a volumetric array of single-walled semiconductor carbon nanotubes (CNTs) embedded in a homogeneous dielectric medium. The nanotubes considered here are of the "zigzag" type $(m, 0)$, where integer $m$ (not a multiple of three for semiconductor nanotubes) determines their radius, $R=m b \sqrt{3} / 2 \pi$, with $b=1.42 \times 10^{-8} \mathrm{~cm}$ being the distance between neighboring carbon atoms [4-7]. The CNTs are arranged in such a way that their axes are parallel to the common $x$ axis, and distances between adjacent nanotubes are much larger than their diameter. The latter assumption allows one to neglect the interaction between CNTs [39]. Moreover, it allows us to consider the system as an electrically quasi-1D one, in which electron tunneling between neighboring nanotubes may be neglected, and electrical conductivity is possible only along the axis of the nanotubes. We define the configuration of the system in such a way that the pulse propagates through the CNT array in the direction perpendicular to their axes (for the sake of definiteness, along the $z$ axis), while the electric component of the wave field, $\mathbf{E}=\{E, 0,0\}$, is collinear with the $x$ axis (see Fig. 1).

For a wide range of values of the system's parameters, the characteristic distance at which an appreciable change in the field of a bipolar electromagnetic pulse occurs is significantly greater than both the distance between neighboring nanotubes and the length of the conduction-electron's path along the axis of the nanotubes. On the other hand, with the nanotube radius $R \approx 5.5 \times 10^{-8} \mathrm{~cm}$ and $m=7$, the characteristic distance between the nanotubes, sufficient to exclude the overlap of the electron wave functions of adjacent ones, i.e., substantially exceeding $R$, may still be negligibly small in comparison with the wavelength $\lambda$ of the electromagnetic radiation. In particular, this assumption is definitely valid for the infrared radiation, with $\lambda>1 \mu \mathrm{m}$. Under this condition, the nanotube array,

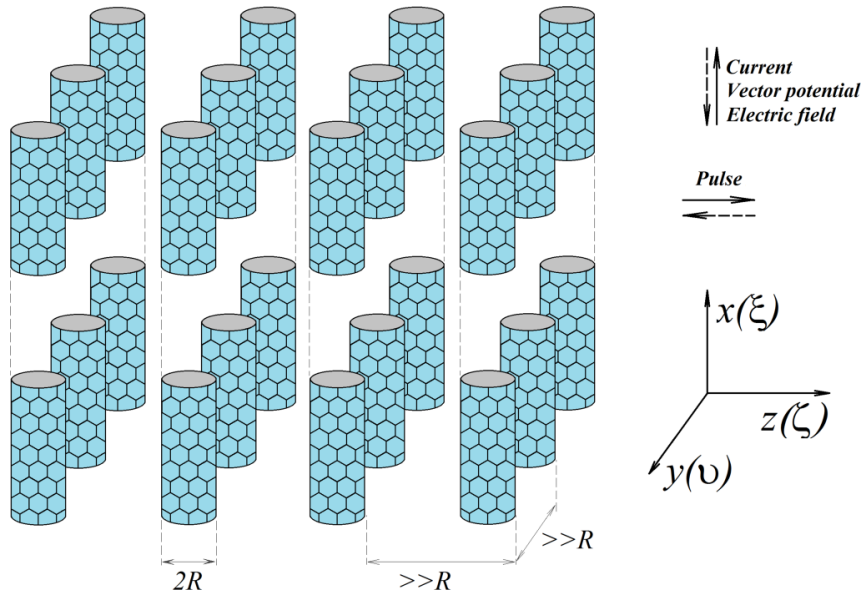

FIG. 1. The schematic plot of the setup and the associated coordinate system.

which is a discrete structure at the microscopic level, may nevertheless be considered as a quasiuniform medium for the propagation of electromagnetic waves. In this case, for length scales comparable to the pulse's dimensions, the array of CNTs may be considered as a uniform continuous medium. In other words, the electromagnetic field in the system-specifically, ultrashort pulses carried by the infrared wavelength-are not affected by the discrete structure in the medium, there being no scattering (or even recurrent scattering) of electromagnetic radiation on inhomogeneities or irregularities of the CNT volumetric array. Of course, the scattering will appear in the framework of a microscopic theory (see, e.g., Ref. [40]), which should be a subject for a separate work.

Another assumption that we adopt here concerns the time duration of the electromagnetic pulse, $T_{S}$, the relaxation time of the conduction current along the nanotube axis, $t_{\text {rel }}$, and also the time of the observation of the light propagation in the system, $t$-we assume $T_{S} \ll t<t_{\text {rel }}$. If this condition is met, it is possible to neglect field decay, thus enabling the collisionless approximation in describing the lossless evolution of the field [12].

\section{GOVERNING EQUATIONS}

Given the orientation of the coordinate system relative to the nanotube axis, as defined in Fig. 1, the electron energy spectrum for CNTs takes the form $[31,32]$

$\epsilon\left(p_{x}, s\right)=\gamma_{0} \sqrt{1+4 \cos \left(p_{x} \frac{d_{x}}{\hbar}\right) \cos \left(\pi \frac{s}{m}\right)+4 \cos ^{2}\left(\pi \frac{s}{m}\right)}$,

where the electron quasimomentum is $\mathbf{p}=\left\{p_{x}, s\right\}$, where $s=$ $1,2, \ldots, m$ is an integer characterizing the momentum quantization along the perimeter of the nanotube, $m$ is the number of hexagonal carbon cycles which form the circumference of the CNT, $\gamma_{0}$ is the overlap integral, and $d_{x}=3 b / 2$.

\section{A. Equation for the vector potential}

The electromagnetic field in the CNT array is governed by Maxwell's equations [41,42], from which, taking into 
account the Lorentz gauge condition, we obtain the wave equation for the spatiotemporal evolution of the vector field potential,

$$
\frac{\varepsilon}{c^{2}} \frac{\partial^{2} \mathbf{A}}{\partial t^{2}}-\frac{\partial^{2} \mathbf{A}}{\partial x^{2}}-\frac{\partial^{2} \mathbf{A}}{\partial y^{2}}-\frac{\partial^{2} \mathbf{A}}{\partial z^{2}}=\frac{4 \pi}{c} \mathbf{j}
$$

with $\mathbf{A}=\{A, 0,0\}$, where $\mathbf{j}=\{j, 0,0\}$ is the current density, $c$ is the speed of light in vacuum, and $\varepsilon$ is the average relative dielectric constant of the medium (see, e.g., Refs. [27,29-32]).

We emphasize that the system under consideration has a nonzero electric conductivity only along the $x$ axis, while in the $(y, z)$ plane, the current is absent due to the negligible coupling between neighboring nanotubes. Thus, since the second and third components of the conduction current $\mathbf{j}$ are zero, Eq. (2) admits the existence of zero solutions for the second and third components of the vector potential. We use this fact to define the vector potential as being collinear to axes of the nanotubes.

The conduction current density $j$ along the nanotube axis is determined by applying the approach used in Refs. [43,44], which yields

$$
j=2 e \sum_{s=1}^{m} \int_{-\pi \hbar / d}^{\pi \hbar / d} v_{x} f\left(p_{x}, s\right) d p_{x},
$$

where $e<0$ is the electron charge, $v_{x}$ is the electron velocity, and $f\left(p_{x}, s\right)$ is the electron distribution function with respect to quasimomenta $p_{x}$ and numbers $s$ characterizing the quantization of the electron's momentum along the perimeter of a nanotube. The integration over the quasimomentum in Eq. (3) is carried out within the first Brillouin zone.

Using the expression for the electron energy (1) to determine their velocity as $v_{x}=\partial \epsilon\left(p_{x}, s\right) / \partial p_{x}$, and taking into account the electron distribution $f\left(p_{x}, s\right)$ according to the Fermi-Dirac statistics, we derive from Eq. (3) an expression for the current density (for more details, see Ref. [32]),

$$
j=-e n \frac{d_{x}}{\hbar} \gamma_{0} \sum_{r=1}^{\infty} G_{r} \sin \left[r \frac{d_{x}}{\hbar}\left(A \frac{e}{c}+e \int_{0}^{t} \frac{\partial \phi}{\partial x} d t^{\prime}\right)\right],
$$

where $n=n(x, y, z, t)$ is the local value of the concentration of conduction electrons, $\phi$ is the scalar potential (self-consistent equations for the quantities $n$ and $\phi$ are derived in Secs. III B and III C, respectively), and coefficients $G_{r}$ are given by

$$
G_{r}=-r \frac{\sum_{s=1}^{m} \frac{\delta_{r, s}}{\gamma_{0}} \int_{-\pi}^{+\pi} \cos (r \kappa)\left\{1+\exp \left[\frac{\theta_{0, s}}{2}+\sum_{q=1}^{\infty} \theta_{q, s} \cos (q \kappa)\right]\right\}^{-1} d \kappa}{\sum_{s=1}^{m} \int_{-\pi}^{+\pi}\left\{1+\exp \left[\frac{\theta_{0, s}}{2}+\sum_{q=1}^{\infty} \theta_{q, s} \cos (q \kappa)\right]\right\}^{-1} d \kappa} .
$$

Here, $\theta_{r, s}=\delta_{r, s}\left(k_{B} T\right)^{-1}$, while $T$ is the temperature, $k_{B}$ is the Boltzmann constant, and $\delta_{r, s}$ are coefficients of the Fourier decomposition [45] of spectrum (1),

$$
\delta_{r, s}=\frac{d_{x}}{\pi \hbar} \int_{-\pi \hbar / d_{x}}^{-\pi \hbar / d_{x}} \epsilon\left(p_{x}, s\right) \cos \left(r \frac{d_{x}}{\hbar} p_{x}\right) d p_{x} .
$$

The evolution of the vector potential of the field in the system is determined by the projection of Eq. (2) onto the nanotube axis, which, taking into account expression (4) and after introducing dimensionless variables, takes the following form:

$$
\begin{aligned}
& \frac{\partial^{2} \Psi}{\partial \tau^{2}}-\left(\frac{\partial^{2} \Psi}{\partial \xi^{2}}+\frac{\partial^{2} \Psi}{\partial v^{2}}+\frac{\partial^{2} \Psi}{\partial \zeta^{2}}\right) \\
& +\eta \sum_{r=1}^{\infty} G_{r} \sin \left[r\left(\Psi+\int_{0}^{\tau} \frac{\partial \Phi}{\partial \xi} d \tau^{\prime}\right)\right]=0,
\end{aligned}
$$

where $\eta=n / n_{\text {bias }}=\eta(\xi, v, \zeta, \tau)$ is the reduced (dimensionless) density of the conduction electron, $n_{\text {bias }}$ is the concentration of conduction electrons in the homogeneous part of the sample in the absence of electromagnetic fields, $\Psi=$ $\operatorname{Aed}_{x} /(c \hbar)$ is the projection of the scaled vector potential onto the $x$ axis, $\Phi=\phi \sqrt{\varepsilon} e d_{x} /(c \hbar)$ is the dimensionless scalar potential, $\tau=\omega_{0} t / \sqrt{\varepsilon}$ is the scaled time, $\xi=x \omega_{0} / c$, $v=y \omega_{0} / c$, and $\zeta=z \omega_{0} / c$ are the scaled coordinates, and

$$
\omega_{0} \equiv 2 \frac{|e| d_{x}}{\hbar} \sqrt{\pi \gamma_{0} n_{\text {bias }}} .
$$

Thus, Eq. (7) describes the evolution of the vector potential of the self-consistent electromagnetic field in the CNT array: the field is fully determined by the density of the conduction current [see Eq. (2)] and the conduction current is, in turn, affected by the field [see Eq. (4)].

\section{B. Equation for the electron density}

In the general case, the electromagnetic field in the system under consideration is nonuniform in space. Indeed, the field of an ultrashort electromagnetic pulse propagating in an array of nanotubes is localized at each moment in a small (moving) region of space. The nonuniformity is invisible on the scale of the nanotube radius, $\sim 5 \times 10^{-8} \mathrm{~cm}$, or even for the distance between neighboring nanotubes, $\sim 10^{-7}-10^{-6} \mathrm{~cm}$. However, it is significant at the wavelength scales of the infrared radiation, $\lambda \sim 1 \mu \mathrm{m}$.

The spatial nonuniformity of the field along the nanotube axis determines the dependence of the current density on coordinate $x$, as it follows from expression (4) for the current density. Since the total charge in the sample is conserved and the change in its bulk density $\rho=e n$ obeys the continuity equation $\nabla \mathbf{j}+\partial \rho / \partial t=0[41,42]$, the nonuniformity of the current density causes a temporal change in electron density, as per

$$
\frac{\partial n}{\partial t}=-\frac{1}{e} \frac{\partial j}{\partial x}
$$

We stress that as the system considered here is supposed to be electrically quasi-one dimensional, i.e., the conductivity is only effective along the nanotubes axis, given the negligible overlap of the electron wave functions of neighboring nanotubes, 
the field nonuniformity along directions orthogonal to the nanotube axes does not affect the distribution of the electron concentration in the sample.

Substituting Eq. (4) for the projection of the current density onto the axis of nanotubes into Eq. (9) and passing to the dimensionless notation (the same as in Ref. [32]), we obtain an equation governing the evolution of the electron concentration under the action of the electromagnetic pulse,

$$
\frac{\partial \eta}{\partial \tau}=\alpha \sum_{r=1}^{\infty} G_{r} \frac{\partial}{\partial \xi}\left\{\eta \sin \left[r\left(\Psi+\int_{0}^{\tau} \frac{\partial \Phi}{\partial \xi} d \tau^{\prime}\right)\right]\right\},
$$

with $\alpha \equiv d_{x} \gamma_{0} \sqrt{\varepsilon} / c \hbar$, and the other quantities defined in Eq. (7).

\section{Equation for the scalar potential field}

Since the system as a whole is electroneutral, the redistribution of the electron concentration in the sample, due to the action of the nonuniform field along the axis of the nanotubes, is equivalent to appearance of regions of high and low electron concentration relative to the initial equilibrium distribution, $n_{0}=n\left(\xi, v, \zeta, \tau_{0}\right)$, taken (at the the initial time $\tau_{0}$ ) prior to the entrance of the electromagnetic pulse into the sample. Thus, the local concentration of electrons, $n(\xi, v, \zeta, \tau)$, may be represented as the sum of the initial equilibrium value $n_{0}$ and the concentration of the "additional" charge, $\delta n(\xi, v, \zeta, \tau)=n-n_{0}$, with density $\delta \rho=e \delta n=e\left(n-n_{0}\right)$. Note that $\delta \rho \neq 0$ implies a local imbalance between the negative charge of free electrons and the positive charge of holes. The local imbalanced charge perturbs the distribution of the field according to the driven wave equation for the scalar potential, which follows from Maxwell's equations [41,42],

$$
\frac{\varepsilon}{c^{2}} \frac{\partial^{2} \phi}{\partial t^{2}}-\left(\frac{\partial^{2} \phi}{\partial x^{2}}+\frac{\partial^{2} \phi}{\partial y^{2}}+\frac{\partial^{2} \phi}{\partial z^{2}}\right)=\frac{4 \pi}{\varepsilon} \delta \rho .
$$

Using the same dimensionless notations as above, Eq. (11) can be written as

$$
\frac{\partial^{2} \Phi}{\partial \tau^{2}}-\left(\frac{\partial^{2} \Phi}{\partial \xi^{2}}+\frac{\partial^{2} \Phi}{\partial v^{2}}+\frac{\partial^{2} \Phi}{\partial \zeta^{2}}\right)=\beta\left(\eta-\eta_{0}\right)
$$

where $\beta=1 / \alpha=c \hbar /\left(d_{x} \gamma_{0} \sqrt{\varepsilon}\right)$ [see Eq. (10)], and $\eta_{0}=$ $n_{0} / n_{\text {bias }}=\eta\left(\xi, v, \zeta, \tau_{0}\right)$ is the dimensionless local value of the concentration of conduction electrons at the initial instant of time in the absence of the field.

Thus, the evolution of the field in the CNT array, taking into account the redistribution of the conduction-electron density, is governed by the system of equations (7), (10), and (12), which provides a self-consistent model for the evolution of the electromagnetic field and electronic subsystem in the array.

\section{The localization of the electromagnetic pulse}

Upon obtaining the numerical solution to Eqs. (7), (10), and (12), it is possible to calculate the electric field as $\mathbf{E}=-c^{-1} \partial \mathbf{A} / \partial t-\nabla \phi$ (see, e.g., Refs. [41,42]). Taking into account that the vector potential has a nonzero component only along the nanotube axis (see the description of the system configuration above), one can write expressions for the components of the electric field as follows:

$$
E_{x}=E_{0}\left(\frac{\partial \Psi}{\partial \tau}+\frac{\partial \Phi}{\partial \xi}\right), \quad E_{y}=E_{0} \frac{\partial \Phi}{\partial v}, \quad E_{z}=E_{0} \frac{\partial \Phi}{\partial \zeta}
$$

where $E_{0} \equiv-\hbar \omega_{0} / e d_{x} \sqrt{\varepsilon}$. Thus, the electric field in the CNT array is not, generally, collinear with the nanotube axis. However, the $y$ and $z$ components of the electric field, orthogonal to the nanotube axis, do not affect the dynamics of electrons due to the absence of conductivity of the system in these directions. Thus, only component $E_{x}$, which affects the dynamics of the electronic subsystem, is relevant to the description of the solitary electromagnetic wave. The energy density of this component is

$$
I=E_{x}^{2}=I_{0}\left(\frac{\partial \Psi}{\partial \tau}+\frac{\partial \Phi}{\partial \xi}\right)^{2}
$$

where $I_{0}=E_{0}^{2}$, and we have made use of the first expression in Eq. (13). The position of a local maxima of this quantity identifies the instantaneous location of the ultrashort electromagnetic pulse.

\section{NONUNIFORMITY OF THE ELECTRON CONCENTRATION}

We assume that the CNT array contains a localized inhomogeneity, in the form of a region with an increased concentration of conduction electrons, exceeding the bulk concentration $n_{\text {bias }}$ in the homogeneous sample. As mentioned above, such a local defect can be created by introducing donor impurities at the stage of the fabrication of the sample, subject to the condition of the electroneutrality of the entire system. Accordingly, the initial electron density (in the absence of an electromagnetic pulse) is $n_{0}=n\left(\xi, v, \zeta, \tau_{0}\right)$. We stress that in the absence of the electromagnetic pulse, each segment of the sample is locally electroneutral, even if the electron concentration is inhomogeneous. Namely, in the region of increased electron density, the hole concentration is higher too, compensating for the charge of the free electrons.

We assume that the region of the increased electron concentration is a narrow layer parallel to the nanotube axis, and its thickness $\delta z_{\text {imp }}$ is much smaller than the spatial dimension of the electromagnetic pulse in the direction of its propagation along the $z$ axis. In addition, we also consider this narrow layer as being indefinitely extended in the $x$ and $y$, as shown in Fig. 2 .

The respective dimensionless electron concentration in the sample in the absence of the electromagnetic field is approximated by the Gaussian profile (see Ref. [37]),

$$
\eta\left(\xi, v, \zeta, \tau_{0}\right)=\eta(\zeta)=1+\left(\eta_{\mathrm{imp}}^{\max }-1\right) \exp \left\{-\left(\frac{\zeta}{\delta \zeta_{\text {imp }}}\right)^{2}\right\}
$$

where $\eta_{\mathrm{imp}}^{\max }=n_{\mathrm{imp}}^{\max } / n_{\text {bias }}, n_{\mathrm{imp}}^{\max }$ is the maximum electron concentration in the region of inhomogeneity, and $\delta \zeta_{\text {imp }}$ is a dimensionless parameter determined by the characteristic half thickness of the region of the increased electron concentration, $\delta \zeta_{\text {imp }}=\omega_{0} \delta z_{\text {imp }} / c$. The concentration of conduction electrons is assumed to be constant in the $(x, y)$ plane. 


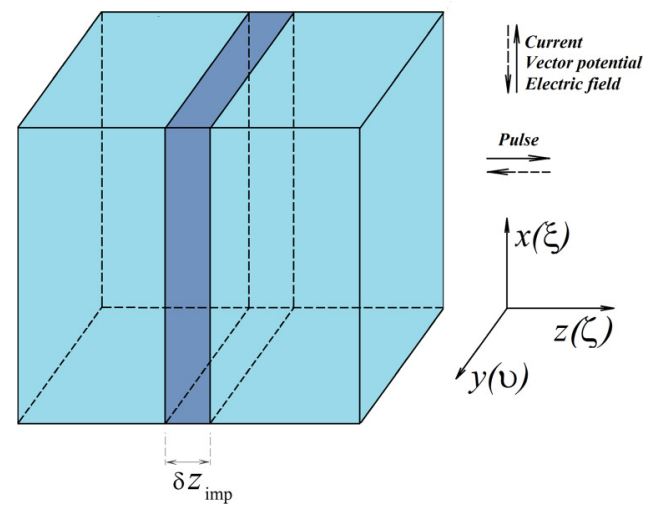

FIG. 2. The layer of increased concentration of conduction electrons.

The initial electron density in the system determines the corresponding scalar potential. Taking into account the fact that $\eta \equiv \eta_{0}$ holds initially, the right-hand side of Eq. (12) vanishes. As a result, Eq. (12) produces a constant solution. Since the scalar potential is always determined up to an arbitrary constant $[41,42]$, its initial value may be fixed to be zero,

$$
\Phi\left(\xi, v, \zeta, \tau_{0}\right)=0
$$

which we assume to be the initial distribution of the scalar potential in the system.

\section{THE INITIAL FORM OF THE ELECTROMAGNETIC PULSE}

We now assume that the electromagnetic pulse propagates in the array of CNTs, with the $\xi$ component of the dimensionless vector potential at the initial instant of time $\tau=\tau_{0}$ defined as follows:

$$
\Psi\left(\xi, v, \zeta, \tau_{0}\right)=\Psi_{B}\left(\zeta, \tau_{0}\right) \exp \left[-\frac{\left(\xi-\xi_{0}\right)^{2}+\left(v-v_{0}\right)^{2}}{w_{0}^{2}}\right]
$$

where $\Psi_{B}\left(\zeta, \tau_{0}\right)$ is the $\xi$ component at $\xi=\xi_{0}$ and $v=v_{0}$, with $\xi_{0}$ and $v_{0}$ being the dimensionless pulse's coordinates, along the $\xi$ and $v$ axes, respectively, at the initial instant of time, and the initial transverse half-width $w_{0}$ of the pulse.

Profile $\Psi_{B}\left(\zeta, \tau_{0}\right)$ is chosen as a breather of the sine-Gordon equation, i.e., a nontopological oscillating soliton [46],

$$
\Psi_{B}\left(\zeta, \tau_{0}\right)=4 \arctan \left\{\left(\frac{1}{\Omega^{2}}-1\right)^{1 / 2} \frac{\sin \chi}{\cosh \mu}\right\}
$$

where

$$
\chi \equiv \sigma \Omega \frac{\tau_{0}-\left(\zeta-\zeta_{0}\right) U}{\sqrt{1-U^{2}}}
$$

$$
\mu \equiv \sigma\left[\tau_{0} U-\left(\zeta-\zeta_{0}\right)\right] \sqrt{\frac{1-\Omega^{2}}{1-U^{2}}}
$$

$U=u / v_{0}$ is the ratio of the initial propagation velocity $u$ of the electromagnetic pulse (breather) along the $\zeta$ axis within the sine-Gordon approximation, and the linear speed of light in the medium, $v_{0}=c / \sqrt{\varepsilon}$. Further, $\zeta_{0}$ is the dimensionless coordinate of the breather along the $\zeta$ axis at moment $\tau=\tau_{0}, \Omega<1$ is a free parameter, which determines the breather's oscillation frequency (scaled by frequency $\omega_{B}=\omega_{0} \Omega$ in physical units), and $\sigma=\sqrt{G_{1}}$ [coefficients $G_{j}$ are calculated as per Eq. (5)].

The basic argument in favor of the choice of the initial condition in the form of Eqs. (17) and (18) is that the equation for the vector potential (7) may be considered as a nonuniform generalization of the sine-Gordon equation. As the sine-Gordon equation gives rise to breather solutions in the form of Eq. (18), it is reasonable to assume the possibility of the propagation of solitary waves in a form close to breathers. This assumption is amply justified by results reported in Refs. [27-32]. The second factor in Eq. (17) corresponds to the Gaussian distribution of the field in the plane $(\xi, \zeta)$ perpendicular to the propagation direction of the electromagnetic pulse. The choice of the Gaussian distribution for this field is justified by a wide range of applicability of the Gaussian wave forms [10,47-50].

The component of the electric field (13) along the nanotube axis, taking into account the expression (17) at the initial instant of time, has the form of

$$
E_{x}=4 E_{0} \frac{\sigma \sqrt{1-\Omega^{2}}}{\sqrt{1-U^{2}}}\left\{\frac{\cos \chi \cosh \mu-U\left(\Omega^{-2}-1\right)^{1 / 2} \sin \chi \sinh \mu}{\cosh ^{2} \mu+\left(\Omega^{-2}-1\right) \sin ^{2} \chi}\right\} \exp \left[-\frac{\left(\xi-\xi_{0}\right)^{2}+\left(v-v_{0}\right)^{2}}{w_{0}^{2}}\right] .
$$

Equations (17)-(21) describe a short wave packet, consisting of a carrier wave and an envelope. The carrier, which accounts for the internal oscillations [27,31] of the pulse, is determined by the oscillating behavior of the function $\sin \chi$, while the envelope accounts for the exponential behavior of the function $\cosh \mu$. In the case of a few-cycle pulse, variation scales of both the envelope and carrier of the pulse have the same order of magnitude, and hence its profile varies periodically with the frequency of the carrier. The pulse given by Eq. (21) is categorized as a "bipolar" one, as the sign of this field component changes periodically.
We emphasize that the initial parameters of the electromagnetic pulse at $\tau=\tau_{0}$ are given under the assumption that the pulse is still located at a sufficient distance from the inhomogeneity layer, where the scaled concentration of conduction electrons (15) is different from 1 . From the experimental viewpoint, the effective optical frequency $\omega_{\mathrm{opt}}$ and the characteristic duration of the pulse, $T_{S}$, are relevant parameters characterizing the shape of the electromagnetic pulse [37].

The optical frequency $\omega_{\text {opt }}$ is determined as follows. As said above, the internal vibrations of the breather (18) are represented by the function $\sin \chi$. We represent the argument 
[see Eq. (19)] of this function in the form

$$
\chi=\omega_{c} t_{0}+k_{\text {wave }}\left(z-z_{0}\right),
$$

where $k_{\text {wave }}$ is the wave vector. In dimensionless form and using Eq. (19), we have

$$
\chi=\frac{\omega_{0}}{\sqrt{\varepsilon}} \frac{\sigma \Omega}{\sqrt{1-U^{2}}} t_{0}-U \frac{\sigma \Omega}{\sqrt{1-U^{2}}}\left(z-z_{0}\right) .
$$

By comparing Eqs. (19) and (20), we obtain the following expression for the carrier-wave frequency:

$$
\omega_{c}=\frac{\omega_{0}}{\sqrt{\varepsilon}} \frac{\sigma \Omega}{\sqrt{1-U^{2}}} .
$$

It must, however, be noticed that frequency $\omega_{c}$ coincides with the frequency at which the optical spectrum reaches its maximum in the slowly-varying-envelope approximation only. For few- and single-cycle pulses, the latter frequency is larger than the former, with the ratio between them increasing as the number of cycles decreases, up to $\approx 1.66$. Further, due to the strong nonlinear behavior, the spectrum is not conserved in the course of the propagation, and the frequency at which it reaches its maximum oscillates. According to Eqs. (17)-(21), i.e., in the framework of the sine-Gordon approximation, the amplitude of the oscillations can reach $\pm 0.14 \omega_{c}$.

Duration $T_{S}$ of the electromagnetic pulse (wave packet) with vector potential (18) is determined by the factor $\cosh \mu$. The usual FWHM definition of $T_{S}$ is the time during which the instantaneous amplitude of the "running" envelope, measured at a fixed point, exceeds half of its peak value. In the fewcycle regime, the definition of $T_{S}$ should be implemented numerically, using, e.g., the standard deviation. Further, even this standard definition can give rise to ambiguities, as there is some discrepancy between the durations of fields $\Psi$ and $E_{x}$, the ratio of which may become 1.3 in the single-cycle regime.

Therefore, it is more convenient to define the pulse duration in terms of the slowly-varying-envelope approximation. According to the definition (20) of $\mu$, the role of the characteristic normalized duration of the pulse may be played by

$$
\tau_{S}=\frac{1}{\sigma U} \sqrt{\frac{1-U^{2}}{1-\Omega^{2}}} .
$$

The fact that $\tau_{S} \sim 1 / U$ at $U \rightarrow 0$ corresponds to the slowlyvarying-envelope approximation limit, with the expression for the pulse in this limit being

$$
\begin{aligned}
E_{x B}= & E_{0} \frac{\partial \Psi_{B}}{\partial \tau}=4 E_{0} \frac{\sigma \sqrt{1-\Omega^{2}}}{\sqrt{1-U^{2}}} \\
& \times\left\{\frac{\cos \chi \cosh \mu}{\cosh ^{2} \mu+\left(\Omega^{-2}-1\right) \sin ^{2} \chi}\right\},
\end{aligned}
$$

at $\xi=\xi_{0}, \zeta=\zeta_{0}$. It is close to the usual sech-shaped pulse, which would be obtained by neglecting the term $\sin ^{2} \chi$ in the denominator,

$$
E_{x B \operatorname{sech}}=4 E_{0} \frac{\sigma \sqrt{1-\Omega^{2}}}{\sqrt{1-U^{2}}} \frac{\cos \chi}{\cosh \mu},
$$

but does not coincide with it. Furthermore, an explicit result for the envelope does not follow straightforwardly from Eq. (26). Therefore, we opt to define the pulse's width in terms of the sech approximation (27). In this case, the ratio between the FWHM duration, $T_{S}$, and the half width at the maximum value of the sech function, which is precisely $\tau_{S}$ in normalized form, is well known to yield the $2 \ln (2+\sqrt{3})$ factor.

Based on these considerations, we obtain the following duration of the electromagnetic pulse:

$$
T_{S}=2 \ln (2+\sqrt{3}) \frac{\sqrt{\varepsilon}}{\omega_{0} \sigma U} \sqrt{\frac{1-U^{2}}{1-\Omega^{2}}} .
$$

To summarize, the pulse's shape is fully characterized by the dimensionless speed $U$ and frequency of internal oscillations $\Omega$, which determine the carrier frequency $\omega_{c}$ and characteristic duration $T_{S}$.

The number of cycles in the pulse can then be defined as $N_{p}=T_{S} / T_{c}$, where $T_{c}=2 \pi / \omega_{c}$ is the period of the carrier wave. Then, according to Eqs. (24) and (28),

$$
N_{p}=\frac{\ln (2+\sqrt{3}) \Omega}{\pi \sqrt{1-\Omega^{2}} U} .
$$

It is thus seen that the parameter $U$ essentially defines the fewor subcycle character of the pulse, from the slowly-varyingenvelope approximation at $U \rightarrow 0$ to the deeply subcycle configuration at $U \rightarrow 1$. Due to the above-mentioned discrepancy between $\omega_{c}$ and the actual maximum of the optical spectrum, $N_{p}$ defined as per Eq. (29) underestimates the number of optical cycles by approximately $8 \%$ in the limit of the singlecycle regime $\left(N_{p}=1\right)$, which is obtained for $U=0.242$. At $U \rightarrow 1, N_{p}$ approaches 0.24 , while the value computed from the maximum of the optical spectrum is 0.46 . Obviously, the concept of the number of cycles is ambiguous in the subcycle regime.

In the subcycle regime, with $T_{S}<T_{c}$, the oscillations of $\sin \chi$ can no longer be considered as a carrier wave. Then, the central frequency of the pulse is mainly determined by the inverse $1 / T_{S}$ of its duration, as can be checked by numerically computing the optical spectrum, which amounts to the computation of the Fourier transform of $E_{x}$. Hence, we define the optical frequency as $\omega_{\mathrm{opt}}=\omega_{c}$ if $N_{p}>1$, i.e., if $U<0.242$, and $\omega_{\text {opt }}=2 \pi / T_{S}$ otherwise. The corresponding wavelength in vacuum is $\lambda_{\text {opt }}=c T_{c}$ for $U<0.242$ and $\lambda_{\text {opt }}=c T_{S}$ for $U>0.242$. It can easily be checked that the maximum value of field $E_{x}$ is

$$
E_{\max }=4 E_{0} \frac{\sigma \sqrt{1-\Omega^{2}}}{\sqrt{1-U^{2}}},
$$

which increases with $U$ and diverges at $U \rightarrow 1$; hence, $U$ may be also considered as a measure of the pulse's peak intensity $I_{p}$. Within the slowly-varying-envelope approximation,

$$
I_{p}=\frac{c \sqrt{\epsilon}}{8 \pi} E_{\max }^{2}=\frac{2 c \sqrt{\epsilon} E_{0}^{2}}{\pi} \frac{\sigma^{2}\left(1-\Omega^{2}\right)}{1-U^{2}} .
$$

Although the actual intensity depends on the wave's velocity and may differ from this expression, we will use Eq. (31) in the few- and subcycle regimes.

\section{TRANSMISSION AND REFLECTION COEFFICIENTS}

As a result of the interaction with the layer of increased electron concentration, the initial electromagnetic pulse is 
generally split into reflected and transmitted ones. The ratio of the energies of the transmitted and reflected wave packets depends on various parameters, including the initial characteristics of the incident pulse, and also parameters of the scattering layer. To quantify this, we calculate the transmission and reflection coefficients, $K_{\text {pass }}$ and $K_{\text {refl }}$, as per [37],

$$
\begin{aligned}
K_{\mathrm{pass}}= & \frac{\int_{0}^{+\infty} d \zeta \int_{-\infty}^{+\infty} d v \int_{-\infty}^{+\infty} d \xi I\left(\xi, v, \zeta, \tau_{\infty}\right)}{\int_{-\infty}^{+\infty} d \zeta \int_{-\infty}^{+\infty} d v \int_{-\infty}^{+\infty} d \xi I\left(\xi, v, \zeta, \tau_{\infty}\right)} \\
K_{\mathrm{refl}}= & \frac{\int_{-\infty}^{0} d \zeta \int_{-\infty}^{+\infty} d v \int_{-\infty}^{+\infty} d \xi I\left(\xi, v, \zeta, \tau_{\infty}\right)}{\int_{-\infty}^{+\infty} d \zeta \int_{-\infty}^{+\infty} d v \int_{-\infty}^{+\infty} d \xi I\left(\xi, v, \zeta, \tau_{\infty}\right)}
\end{aligned}
$$

In Eqs. (32) and (33), $\tau_{\infty}$ corresponds to any time after the establishment of the stable propagation of the electromagnetic pulse, after its interaction with the layer, when the pulse is already at a sufficiently large distance away from it so that the field energy density in this layer is negligible in comparison to the maximum energy density of the field, i.e., condition $I\left(\xi, v, 0, \tau_{\infty}\right) \ll I_{\max } \equiv \max \left\{I\left(\xi, v, \zeta, \tau_{\infty}\right)\right\}$ holds. Coefficient $K_{\text {pass }}$, defined by Eq. (32), may be interpreted as the ratio of the energy of the wave packet passing the layer of increased electron concentration to the total field energy in the volume of the sample. Similarly, $K_{\text {refl }}$, defined by Eq. (33), is the share of the energy of the reflected wave packet in the sum of the energies of the transmitted and reflected packets.

The system considered in this paper is conservative since the collisionless approximation is assumed. Therefore, the energyconservation law imposes the constraint

$$
K_{\text {refl }}+K_{\text {pass }}=1 .
$$

Note that when condition $I\left(\xi, v, 0, \tau_{\infty}\right) \ll I_{\max }$ is satisfied, and also by virtue of the energy conservation law, coefficients $K_{\text {pass }}$ and $K_{\text {refl }}$ are time independent.

If the energy of the wave packet propagating in the original direction significantly exceeds the energy of the wave packet reflected from the inhomogeneity layer, i.e., $K_{\text {pass }} \gg K_{\text {refl }}$, we assume that the pulse has passed through this layer. In the opposite case, when the energy of the reflected wave packet significantly prevails over the energy of the transmitted one, i.e., $K_{\text {pass }} \ll K_{\text {refl }}$, we categorize the pulse as reflected. For certain values of the system's parameters-in particular, at some "threshold" value of the initial peak intensity, $I_{p_{\text {thr }}}$, of the incident pulse-it may split into two wave packets with approximately equal energies, which propagate in opposite directions after the interaction with the inhomogeneity layer, thereby corresponding to $K_{\text {pass }} \approx K_{\text {refl }}$.

\section{NUMERICAL ANALYSIS AND DISCUSSION OF THE RESULTS}

\section{A. System parameters}

For modeling, we used the following realistic values of the parameters of CNTs of the zigzag type $(m, 0): m=7$, $\gamma_{0}=2.7 \mathrm{eV}, b=1.42 \times 10^{-8} \mathrm{~cm}, d_{x} \approx 2.13 \times 10^{-8} \mathrm{~cm}$, and $n_{\text {bias }}=10^{18} \mathrm{~cm}^{-3}$. We assume that the CNT array is embedded in a dielectric matrix and the resulting effective dielectric constant of the system is $\varepsilon=4$, where the calculation of coefficients $G_{r}$ given by Eq. (5) in the expression for the conduction current density (4) is performed for temperature $T=293 \mathrm{~K}$ (see, for example, Refs. [32,37]).

We note that the use of the collisionless approximation (which makes it possible to neglect dissipative effects, as mentioned above) is justified when the evolution-time interval is smaller than the relaxation time $t_{\text {rel }}$. In the course of the respective time, $t_{\text {rel }} \simeq 10^{-11} \mathrm{~s}$, the electromagnetic pulse passes distance $z \leqslant c t_{\text {rel }} / \sqrt{\varepsilon} \simeq 0.15 \mathrm{~cm}$.

When simulating the interaction of the pulse with a layer of the increased electron concentration, we vary values of the parameters within a wide range. In particular, parameter $\eta_{\mathrm{imp}}^{\max }=n_{\mathrm{imp}}^{\max } / n_{\text {bias }}$, corresponding to the maximum concentration of electrons in the inhomogeneity layer, ranges from 2 to 100 . Next, the layer's dimensionless thickness $\delta \zeta_{\text {imp }}$ is varied between 0.05 to 0.5 . Parameter $U$ of the electromagnetic pulse approaching the layer is chosen in the interval of $U \in(0.5 ; 0.999)$. We note that at velocities $U<0.5$, in the course of time $\sim t_{\text {rel }}$, the pulse passes a negligible distance, which is much smaller than its own spatial width along the $\zeta$ axis. Velocities corresponding to $U>0.999$ are not considered here because of limitations imposed by the numerical scheme.

Frequency $\Omega$ of the internal vibrations of the breatherlike electromagnetic pulse is varied in the interval of $\Omega \in(0.1 ; 0.9)$. As it decreases, the width of the pulse along the $\zeta$ axis decreases too, although for $\Omega \leqslant 0.5$ the corresponding change of the pulse's profile is insignificant. At $\Omega>0.7$, the width of the pulse becomes comparable to dimensions of the numerical grid, which was chosen in accordance with the characteristic size of real samples of CNT arrays. Lastly, transverse width $w_{0}$ of the pulse is varied between 0.5 and 2.0, which leads to no qualitative difference in the character of the interaction of the electromagnetic pulse with the region of increased electron concentration.

\section{B. Scenarios for the interaction of the electromagnetic pulse with a layer of increased electron concentration}

Equations (7), (10), and (12) do not admit analytical solutions, therefore we carried out numerical simulations to study the propagation of the electromagnetic pulse in the CNT array. To solve this system of equations with initial conditions (15)-(17), we used an explicit finite-difference three-layer scheme of the "cross" type described in Refs. [51-53], which was adapted by us for the 3D model, using the approach developed in Ref. [37]. Here, we do not describe it in detail, as the numerical scheme and the computational algorithm are similar to those presented in a detailed form in Ref. [37] for the $2 \mathrm{D}$ geometry. As a result of the computations, we have found fields $\Psi(\xi, v, \zeta, \tau), \eta(\xi, v, \zeta, \tau)$, and $\Phi(\xi, v, \zeta, \tau)$, and also calculated the distribution of the field energy density at each instant of time, using Eq. (14).

The simulations reveal that depending on the values of certain system's parameters, different scenarios of the interaction of the ultrashort pulse with the layer of increased electron concentration are possible. The pulse may either pass the layer or bounce back from it. Control parameters, whose values determine the result of the interaction, are characteristics of the electromagnetic pulse (in particular, the speed at which it is approaching the inhomogeneity layer) 
(a)

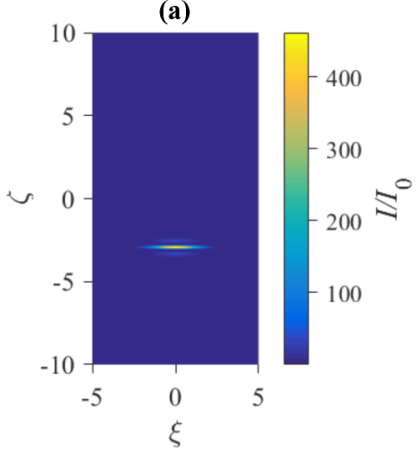

(b)

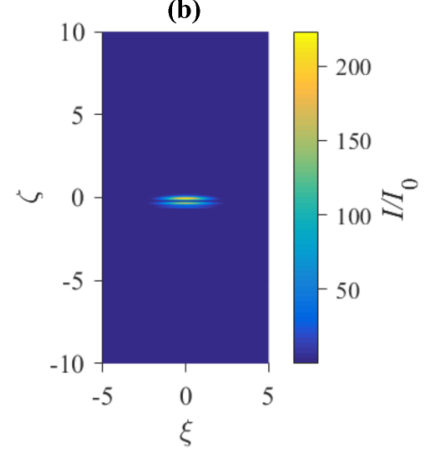

(c)

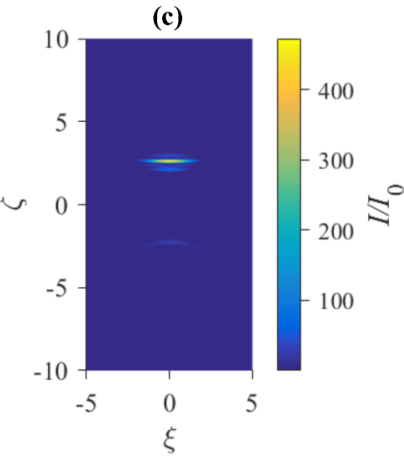

(d)

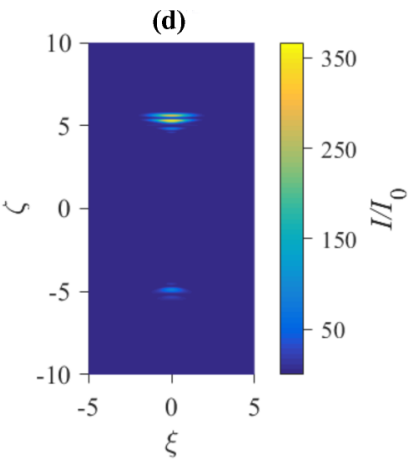

FIG. 3. Distribution of the energy density of the electric field $I\left(\xi, v_{0}, \zeta, \tau\right)$ in the array of nanotubes at various moments of dimensionless time $\tau=\omega_{0} t / \sqrt{\varepsilon}$, in the course of the passage of the laser pulse through the layer of high electron concentration, located at $\zeta=0$ : (a) $\tau=0$, (b) $\tau=3.0$, (c) $\tau=6.0$, (d) $\tau=9.0$. Dimensional coordinates $\xi=x \omega_{0} / c$ and $\zeta=z \omega_{0} / c$ are plotted along the horizontal and vertical axes. Values of $I / I_{0}$ are mapped with the help of the color scale, with yellow and blue areas corresponding, respectively, to the maximum and minimum values of the energy density.

and of the layer itself (its thickness and the concentration of conduction electrons in it). Passing the layer is facilitated both by the increase in the peak intensity of the incident pulse and by the decrease in the thickness of the layer and concentration of electrons in it. In fact, similar scenarios of the interaction of the pulse with the layer are produced by varying all control parameters.

In what follows, we present a number of key results for the propagation of the ultrashort pulse in the inhomogeneous CNT array for different values of parameters of the pulse and inhomogeneity layer. Figures 3 and 4 display the results for the ultrashort pulse interacting with the layer at different values of the pulse's parameter $U$, while other parameters remain constant: the dimensionless frequency of internal oscillations is $\Omega=0.5$, the transverse pulse's width $w_{0}=1.75$, and characteristics of the inhomogeneity layer $\eta_{\text {imp }}^{\max }=30, \delta \zeta_{\text {imp }}=0.1$.

Figure 3 illustrates the passage of the electromagnetic pulse through the layer of increased electron concentration. The temporal narrowness of the incident pulse and its peak intensity are determined by $U=0.99$. The duration of such a pulse is $T_{S} \simeq 12.8 \times 10^{-15} \mathrm{~s}$ [see Eq. (28)], which corresponds to the optical frequency $\omega_{\text {opt }} \simeq 4.9 \times 10^{14} \mathrm{~s}^{-1}$ and wavelength $\lambda_{\text {opt }} \simeq 2.8 \mu \mathrm{m}$, at the lower limit of the midinfrared band. The maximum amplitude of the electric field of the pulse is $E_{\max } \simeq 8.6 \times 10^{4}$ statvolt $/ \mathrm{cm} \simeq 2.6 \times 10^{9} \mathrm{~V} / \mathrm{m}$, which follows from Eq. (30). This electric-field amplitude corresponds to a peak intensity $I_{p} \simeq 1.76 \times 10^{19} \mathrm{erg} \mathrm{cm}^{2} \mathrm{~s}^{-1} \simeq 1.76 \mathrm{TW} / \mathrm{cm}^{2}$.

Figure 3 shows the distribution of the energy density of the electric field $I\left(\xi, v_{0}, \zeta, \tau\right)$ [see Eq. (14)] in the cross section $(\xi, \zeta)$ (at $\left.v=v_{0}\right)$ at different values of the dimensionless time, $\tau=\omega_{0} t / \sqrt{\varepsilon}$. The energy density of the electric field is represented by ratio $I / I_{0}$, using a suitable color map, with blue and yellow areas corresponding, respectively, to minimum and maximum values. For values of the system's parameters selected above, the unit along the $\xi$ and $\zeta$ axes corresponds to distance $\simeq 4.2 \times 10^{-4} \mathrm{~cm}$. Note that we show the distribution of $I / I_{0}$ only in the cross section $(\xi, \zeta)$ (for $v=v_{0}$ ), as the pattern of the distribution of the energy density in the cross section $(v, \zeta)$ is quite similar.

It can be seen from Fig. 3 that the electromagnetic pulse after interacting with the inhomogeneity layer passes it and continues to stably propagate in the medium in the original direction. Note that in this case, only a negligible fraction of the initial electromagnetic pulse is reflected, in the form of a wave packet with a small amplitude propagating in the opposite direction. The transmission and reflection coefficients in this case are $K_{\text {pass }} \approx 0.8464$ and $K_{\text {refl }} \approx 0.1536$, respectively, satisfying relation $K_{\text {pass }} \gg K_{\text {refl }}$, which allows us to speak mainly about the passage of the layer of high electron concentration by the pulse. (a)

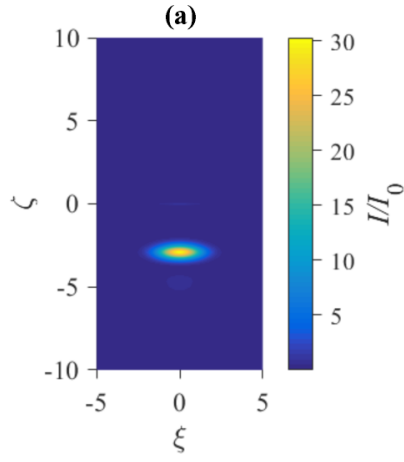

(b)

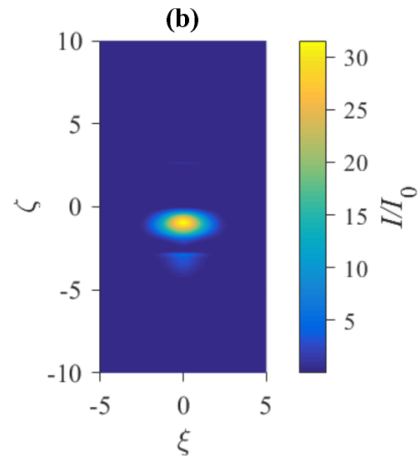

(c)

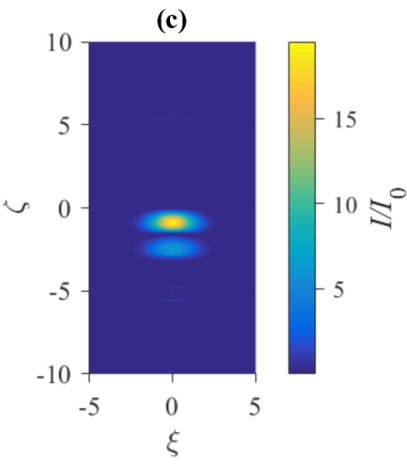

(d)

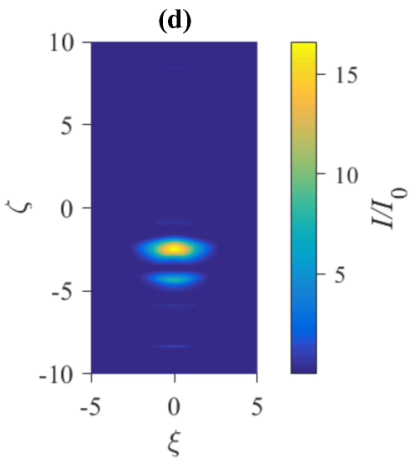

FIG. 4. The distribution of the energy density of the electric field, $I\left(\xi, v_{0}, \zeta, \tau\right)$, in the array of nanotubes at different values of dimensionless time $\tau$, when the laser pulse is reflected from the layer of increased electron concentration placed at $\zeta=0$ : (a) $\tau=0$, (b) $\tau=3.0$, (c) $\tau=6.0$, (d) $\tau=9.0$. The notation is the same as in Fig. 3 . 
(a)

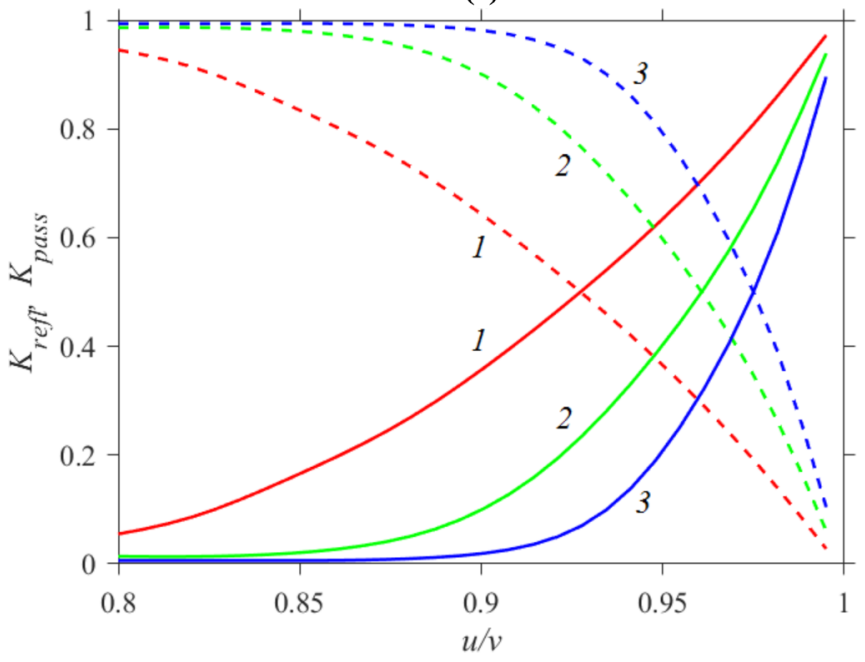

(b)

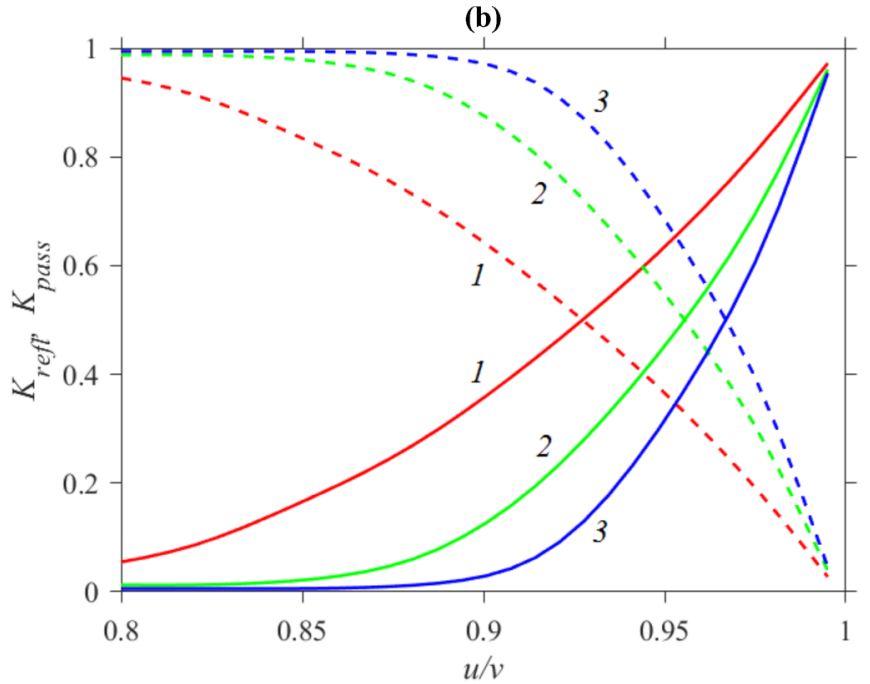

FIG. 5. Transmission and reflection coefficients, $K_{\text {pass }}$ and $K_{\text {refl }}$ (solid and dashed curves, respectively), vs parameter $U$, which characterizes the narrowness and intensity strength of the incident electromagnetic pulse when it interacts with the layer of increased electron concentration (a) for increasing concentration, namely, for $\delta \zeta_{\mathrm{imp}}=0.1: 1$ (red): $\eta_{\mathrm{imp}}^{\max }=20 ; 2$ (green): $\eta_{\mathrm{imp}}^{\max }=30 ; 3$ (blue): $\eta_{\mathrm{imp}}^{\max }=40$; and (b) for increasing values of the layer's thickness, namely, for $\eta_{\mathrm{imp}}^{\max }=20: 1$ (red): $\delta \zeta_{\mathrm{imp}}=0.10 ; 2$ (green): $\delta \zeta_{\text {imp }}=0.15 ; 3$ (blue): $\delta \zeta_{\text {imp }}=0.20$.

Figure 4 shows the reverse situation, namely, the reflection of the electromagnetic pulse, with $U=0.80$, from the layer of increased electron concentration. These values of the parameters of the electromagnetic pulse correspond to duration $T_{S} \simeq$ $6.7 \times 10^{-14} \mathrm{~s}$, optical frequency $\omega_{\text {opt }} \simeq 9.3 \times 10^{13} \mathrm{~s}^{-1}$, and wavelength $\lambda_{\text {opt }} \simeq 20 \mu \mathrm{m}$, in the midinfrared range, and the maximum of the electric field $E_{\max } \simeq 2.0 \times 10^{4}$ statvolt $/ \mathrm{cm} \simeq$ $6.1 \times 10^{8} \mathrm{~V} / \mathrm{m}$, which corresponds to peak intensity $I_{p} \simeq$ $9.75 \times 10^{17} \mathrm{erg} \mathrm{cm}^{2} \mathrm{~s}^{-1} \simeq 97.5 \mathrm{GW} / \mathrm{cm}^{2}$. Similarly to Fig. 3, this figure shows the distribution of the energy density of the electric field $I\left(\xi, v_{0}, \zeta, \tau\right)$ in cross section $(\xi, \zeta)$ of the CNT array at various values of dimensionless time $\tau$. The electromagnetic pulse, having an insufficiently high initial peak intensity, does not pass this region, bouncing back and propagating in the reverse direction. The respective transmission and reflection coefficients are $K_{\text {pass }} \approx 0.075$ and $K_{\text {refl }} \approx$ 0.9925. Relation $K_{\text {pass }} \ll K_{\text {refl }}$ in this case may be interpreted as satisfying the criterion for the reflection of the laser pulse from the layer of an increased electron concentration.

As shown by the numerical analysis, the possibility of the passage of the electromagnetic pulse through the layer of the high electron concentration depends not only on the peak intensity of the incident pulse, but also on parameters of the layer, such as its thickness $\delta \zeta_{\text {imp }}$ and the maximum reduced concentration of electrons in it, $\eta_{\text {imp }}^{\max }$.

Figure 5 shows the dependence of the reflection and transmission coefficients of the electromagnetic pulse on parameter $U$ that determines its initial peak intensity, longitudinal width, and duration. As $U$ increases, the transmission coefficient $K_{\text {pass }}$ (the solid curve) increases too and, accordingly, the reflection coefficient $K_{\text {refl }}$ (the dotted curve) decreases. The value $U=U_{\text {thr }}$ of $U$ is such that the two curves intersect, thereby implying the equality between the transmission and reflection coefficients,

$$
K_{\text {pass }}\left(U_{\text {thr }}\right)=K_{\text {refl }}\left(U_{\text {thr }}\right) .
$$

The latter equation fully defines the threshold value $U_{\mathrm{thr}}$. For values of parameters used in Figs. 3 and 4, namely, $\eta_{\mathrm{imp}}^{\max }=30$ and $\delta \zeta_{\text {imp }}=0.1$, the threshold is $U_{\text {thr }} \simeq 0.96$, which corresponds to the peak intensity $I_{p_{\text {thr }}} \simeq 4.6 \times 10^{18} \mathrm{erg} \mathrm{cm}^{2} \mathrm{~s}^{-1}=$ $460 \mathrm{GW} / \mathrm{cm}^{2}$. At $U \simeq U_{\mathrm{thr}}$, the electromagnetic pulse can be divided into two approximately identical wave packets, one of which continues to move in the original direction, while the other bounces back from the layer of increased electron concentration. Calculations show that the transmission coefficient $K_{\text {pass }}$ increases and the reflection coefficient $K_{\text {refl }}$ decreases with the increase of $U$, while other parameters are kept constant. Further, $K_{\text {pass }}$ decreases and $K_{\text {refl }}$ increases with the increase of any parameter of the inhomogeneity layer, viz., $\eta_{\text {imp }}^{\max }$ [see Fig. 5(a)] and $\delta \zeta_{\text {imp }}$ [see Fig. 5(b)], at a fixed value of $U$.

Thus, the result of the interaction of the laser pulse with the layer of the increased electron concentration depends on various parameters, including the initial pulse's parameter $U$, which controls peak intensity $I_{p}$, optical frequency $\omega_{\text {opt }}$, duration $T_{S}$, the maximum reduced electron concentration in the inhomogeneity layer, $\eta_{\mathrm{imp}}^{\max }$, and the characteristic thickness of this layer, $\delta \zeta_{\text {imp }}$.

Qualitatively, these dependencies have a simple physical explanation. As can be seen from Eq. (4), the current density induced by the pulse is proportional to the conduction electrons' concentration. Therefore, with the increase of the carrier concentration in the impurity band, the current that creates the pulse also increases, i.e., the impurity region becomes more conductive. Even at the level of linearized equations, using the Fresnel formulas for the reflection and transmission coefficients, it is clear that a more conducting medium reflects the electromagnetic wave more efficiently. The obtained dependencies on the speed of the pulses may be qualitatively understood too. As the peak intensity increases, the velocity also increases, reducing the time of the interaction with the impurity band, and hence the time available for generating the pulse running in the reverse direction is reduced. Indeed, as can be seen from Eq. (31), the higher the intensity, the higher the 
pulse velocity is. From Eq. (27), one can notice that a higher pulse velocity leads to a reduction in the longitudinal width of the pulse, which consequently yields a shorter pulse duration [Eq. (28)]. In summary, a decrease in the pulse duration and longitudinal width results in a shorter time interval during which the pulse interacts and passes through the region with an increased electron concentration.

Another explanation for the particular pulse dynamics observed in the presence of the layer of inhomogeneity is that a higher peak intensity of the pulse makes it easier to overcome the effective potential barrier induced by the impurity band. In Refs. [54,55], an energy-based analysis of the interaction between the pulse and a layer of increased electron concentration in a semiconductor is reported. The energy of the soliton, necessary to overcome the effective potential barrier created by the impurity region, was defined through the effective Lagrangian of the soliton in a vicinity of an impurity, in the framework of a model based on an inhomogeneous (perturbed) wave equation of the sine-Gordon type, similar to the governing equation used in the present work. In Refs. [54,55], it was established that larger values of the pulse's velocity — or, equivalently, of the peak intensityand smaller values of the pulse duration favor the passage of a pulse through the region of increased conductivity.

In connection to these results, it is relevant to compare them with known results obtained for the $1 \mathrm{D}$ sine-Gordon equation with impurities [56-59]. Conclusions formulated in those works indicate that colliding with an inhomogeneity, the breather is either attenuated, due to the emission of linear waves, or splits into a kink-antikink pair (note that an essential part of those above results was obtained analytically). The difference demonstrated by our results is related to two central factors: (i) the three dimensionality of our problem, which modifies the dispersion law, and (ii) the nonlinearity of our medium, represented by multiple sine terms. Because of the nonintegrability of the present model, our quasisoliton suffers radiation losses, which, however, become significant for times much larger than those we are considering here, namely, at times when the relaxation effects in the electronic subsystem become significant. The effects of the three dimensionality, in particular, imply the necessity to redefine the topological charge, which plays a major role in the dynamics of the $1 \mathrm{D}$ models. In the $1 \mathrm{D}$ case, the topological charge acts as a "selection rule," which prohibits certain decay mechanisms. In the present case, these rules do not apply because of the three dimensionality. While the breatherlike solutions suffer some radiation losses, as mentioned above, it is not seen in the reflection or transmission coefficients, as the integration is carried out throughout the entire spatial domain, taking the contribution from the radiation field into account.

Thus, based on the results produced by our numerical analysis, it can be asserted that the electromagnetic pulses with relatively low peak intensities cannot pass the layer of increased electron concentration, while the pulses with peak intensities significantly exceeding a certain threshold value overcome the repelling layer. As the peak intensity of the pulse increases, the possibility of its passage through the layer increases too. In other words, an increase in the optical frequency and a decrease in the duration of the electromagnetic pulse contribute to the ability of the pulse to pass the layer.
The threshold value of parameter $U_{\text {thr }}$ of the electromagnetic pulse, in turn, depends on a number of factors, including parameters $\eta_{\text {imp }}^{\max }$ and $\delta \zeta_{\text {imp }}$ of the layer of increased electron concentration. The possibility of the passage of the inhomogeneity layer by the pulse increases with a decrease in these parameters. These findings refine and generalize conclusions obtained in previous works devoted to the study of the interaction of extremely short pulses with layers of an increased electron concentration in CNT arrays, which were based on 2D models [36,37]. The system considered in the present work acts as a "filter" for extremely short electromagnetic pulses, selectively transmitting narrow ones (with higher optical frequencies) and reflecting pulses of longer durations, with lower frequencies. This effect may be used as a basis for the operation of optical logic elements and laser-field control devices, as well as in the technology of nondestructive quality control of electronic elements based on CNTs.

\section{CONCLUSIONS}

Key results of this work are summarized as follows:

(i) It has been established that as a result of the scattering of the electromagnetic pulse on the layer of increased electron concentration in the array of CNTs (carbon nanotubes), both the passage of the pulse through the layer and reflection from it take place.

(ii) The result of the interaction of the electromagnetic pulse with the layer of increased electron concentration depends on values of the system's parameters, including the speed (determined by the optical frequency and duration) of the pulse, and also on characteristics of the inhomogeneity layer (its thickness and the excess of the conduction electron concentration with respect to the bulk array).

(iii) The increase in the peak intensity (or increase in the optical frequency or decrease in the duration) of the electromagnetic pulse, as well as the decrease in the thickness of the inhomogeneity layer and concentration of conduction electrons in it, facilitate the passage of the pulse through this layer.

(iv) After interacting with the layer of high electron concentration, the electromagnetic pulse retains its characteristics, remaining an oscillating bipolar wave packet that can propagate steadily over distances that are noticeably larger than its dimensions along the direction of motion.

\section{ACKNOWLEDGMENTS}

A.V.Z. and R.B. are financially supported by the SUTDMIT International Design Centre (IDC). N.N.R. acknowledges the support from the Russian Foundation for Basic Research, Grant No. 16-02-00762, and from the Foundation for the Support of Leading Universities of the Russian Federation (Grant No. 074-U01). M.B.B. acknowledges support from the Russian Foundation for Fundamental Research. E.G.F. is grateful to Professor Tom Shemesh for his generous support. B.A.M. appreciates the hospitality of the School of Electrical and Electronic Engineering at the Nanyang Technological University (Singapore).

All the authors contributed equally to this work. 
[1] R. H. Baughman, A. A. Zakhidov, and W. A. de Heer, Science 297, 787 (2002).

[2] S. Iijima, Nature (London) 354, 56 (1991).

[3] S. Iijima and T. Ichihashi, Nature (London) 363, 603 (1993).

[4] M. S. Dresselhaus, G. Dresselhaus, and P. Eklund, The Science of Fullerenes and Carbon Nanotubes (Elsevier, New York, 1996).

[5] Carbon Nanotubes, Preparation and Properties, edited by T. W. Ebbesen (CRC Press, Boca Raton, FL, 1996).

[6] R. Saito, G. Dresselhaus, and M. S. Dresselhaus, Physical Properties of Carbon Nanotubes (World Scientific, Singapore, 1998).

[7] P. J. F. Harris, Carbon Nanotubes and Related Structures: New Materials for the Twenty-First Century (Cambridge University Press, Cambridge, 1999).

[8] S. A. Maksimenko and G. Ya. Slepyan, J. Commun. Technol. Electron. 47, 235 (2002).

[9] S. A. Maksimenko and G. Ya. Slepyan, Handbook of Nanotechnology. Nanometer Structure: Theory, Modeling, and Simulation (SPIE, Bellingham, 2004).

[10] A. V. Zhukov, R. Bouffanais, M. B. Belonenko, and E. G. Fedorov, Mod. Phys. Lett. B 27, 1350045 (2013).

[11] M. B. Belonenko and E. G. Fedorov, Russ. Phys. J. 55, 83 (2012).

[12] M. B. Belonenko, E. V. Demushkina, and N. G. Lebedev, J. Russ. Laser Res. 27, 457 (2006).

[13] S. A. Akhmanov, V. A. Vysloukh, and A. S. Chirkin, Optics of Femtosecond Laser Pulses (American Institute of Physics, College Park, MD, 1992).

[14] A. M. Zheltikov, Phys. Usp. 50, 705 (2007).

[15] S. V. Sazonov, Bull. Russ. Acad. Sci.: Phys. 75, 157 (2011).

[16] H. Leblond and D. Mihalache, Phys. Rep. 523, 61 (2013).

[17] G. Mourou, S. Mironov, E. Khazanov, and A. Sergeev, Eur. Phys. J. Spec. Top. 223, 1181 (2014).

[18] M. Kolesik and J. V. Moloney, Rep. Prog. Phys. 77, 016401 (2014).

[19] D. J. Frantzeskakis, H. Leblond, and D. Mihalache, Rom. J. Phys. 59, 767 (2014).

[20] B. A. Malomed, D. Mihalache, F. Wise, and L. Torner, J. Opt. B.: Quantum Semiclass. Opt. 7, R53 (2005).

[21] D. Mihalache, Rom. J. Phys. 57, 352 (2012).

[22] D. Mihalache, Rom. J. Phys. 59, 295 (2014).

[23] M. B. Belonenko, N. N. Yanushkina, and E. G. Fedorov, Bull. Russ. Acad. Sci.: Phys. 76, 1326 (2012).

[24] B. Malomed, L. Torner, F. Wise, and D. Mihalache, J. Phys. B: At. Mol. Opt. Phys. 49, 170502 (2016).

[25] D. Mihalache, Rom. Rep. Phys. 69, 403 (2017).

[26] M. B. Belonenko, N. G. Lebedev, and E. N. Nelidina, Phys. Wave Phen. 19, 39 (2011).

[27] E. G. Fedorov, A. V. Zhukov, M. B. Belonenko, and T. F. George, Eur. Phys. J. D 66, 219 (2012).

[28] H. Leblond and D. Mihalache, Phys. Rev. A 86, 043832 (2012).

[29] E. G. Fedorov, A. V. Pak, and M. B. Belonenko, Phys. Sol. State 56, 2112 (2014).

[30] M. B. Belonenko and E. G. Fedorov, Phys. Sol. State 55, 1333 (2013).
[31] A. V. Zhukov, R. Bouffanais, E. G. Fedorov, and M. B. Belonenko, J. Appl. Phys. 114, 143106 (2013).

[32] A. V. Zhukov, R. Bouffanais, B. A. Malomed, H. Leblond, D. Mihalache, E. G. Fedorov, N. N. Rosanov, and M. B. Belonenko, Phys. Rev. A 94, 053823 (2016).

[33] E. G. Fedorov, N. N. Konobeeva, and M. B. Belonenko, Russ. J. Phys. Chem. B 8, 409 (2014).

[34] M. B. Belonenko, N. G. Lebedev, and A. S. Popov, JETP Lett. 91, 461 (2010).

[35] M. B. Belonenko, A. S. Popov, and N. G. Lebedev, Tech. Phys. Lett. 37, 119 (2011).

[36] A. V. Zhukov, R. Bouffanais, E. G. Fedorov, and M. B. Belonenko, J. Appl. Phys. 115, 203109 (2014).

[37] A. V. Zhukov, R. Bouffanais, H. Leblond, D. Mihalache, E. G. Fedorov, and M. B. Belonenko, Eur. Phys. J. D 69, 242 (2015).

[38] S. V. Kryuchkov and E. V. Kaplya, J. Commun. Technol. Electron 51, 359 (2006).

[39] M. B. Belonenko, S. Yu. Glazov, and N. E. Meshcheryakova, Opt. Spectrosc. 108, 774 (2010).

[40] B. A. van Tiggelen, A. Tip, and A. Lagendijk, J. Phys. A: Math. Gen. 26, 1731 (1993).

[41] L. D. Landau, E. M. Lifshitz, and L. P. Pitaevskii, Electrodynamics of Continuous Media, 2nd ed. (Elsevier, Oxford, 2004).

[42] L. D. Landau and E. M. Lifshitz, The Classical Theory of Fields, 4th ed. (Butterworth-Heinemann, Oxford, 2000).

[43] E. M. Epshtein, Fiz. Tverd. Tela 19, 3456 (1976).

[44] E. M. Epshtein, Fiz. Tech. Polupr. 14, 2422 (1980) [Sov. Phys. Semiconduc. 14, 1438 (1980)].

[45] G. A. Korn and T. M. Korn, Mathematical Handbook for Scientists and Engineers (McGraw Hill, New York, 1968).

[46] Yu. S. Kivshar and B. A. Malomed, Rev. Mod. Phys. 61, 763 (1989).

[47] Femtosecond Laser Pulses: Principles and Experiments, edited by C. Rulliére (Springer-Verlag, Berlin, 1998).

[48] A. N. Pikhtin, Optical and Quantum Electronics (High School Publishers, Moscow, 2001).

[49] A. M. Goncharenko, Gaussian Light Beams (Nauka i Tekhnika, Minsk, 1976).

[50] M. B. Belonenko and E. G. Fedorov, Russ. Phys. J. 55, 436 (2012).

[51] N. N. Kalinkin, Numerical Methods (Nauka, Moscow, 1978).

[52] S. E. Koonin, Computational Physics: Fortran Version (Ingram, Boulder, CO, 1998).

[53] J. W. Thomas, Numerical Partial Differential Equations-Finite Difference Methods (Springer-Verlag, New York, 1995).

[54] S. V. Kryuchkov and E. G. Fyodorov, Laser Phys. 12, 1037 (2002).

[55] S. V. Kryuchkov and E. G. Fyodorov, Opt. Spectrosc. 94, 225 (2003).

[56] B. A. Malomed, Physica D 27, 113 (1987).

[57] B. A. Malomed, Phys. Lett. A 120, 28 (1987).

[58] V. E. Zakharov, S. V. Manakov, S. P. Novikov, and L. P. Pitaevskii, Theory of Solitons (Consultants Bureau, New York, 1984).

[59] Yu. S. Kivshar and B. A. Malomed, Phys. Lett. A 115, 381 (1986). 